\section{VHN 10}

CHEMOTHERAPY AND RADIOTHERAPY (CT+RT) VS.CHEMOTHERAPY ALONE (CT) IN THE TREATMENT OF ADVANCED HEAD AND NECK CANCER (HNC)

L. Bergmann, A. Klima, A. Zoubek, S. Szepesi, C.v. Ilberg, P.S. Mitrou

In advanced HNC, combined madality treatment with chemotherapy followed by surgery and/or radiotherapy (RT) appears to be superior to chemotherapy (CT) or radiotherapy alone. In a prospective randomized study we compared the results of CT+RT (regimen A) to CT alone (regimen $B$ ) in advanced HNC. The administered drugs consisted of cis-platinum $60 \mathrm{mg} / \mathrm{m}^{2}$ (day $1+8$ ), bleomycin $10 \mathrm{mg} / \mathrm{m}^{2}$ (CIVI day $3-7$, i.v.push day $15+22$ ), methotrexate $25 \mathrm{mg} / \mathrm{m}^{2}$ (day $15+22$ ). In regimen $A$ the patients (pts.) received 2 courses of $C T$ followed by irrodiation of the tumor region with $60 \mathrm{gy}$, and in regimen B 3 courses of CT alone was administered. 97 pts.were included into the study. So far $75 / 97 \mathrm{pts}$. were evaluable. All pts. had locally advanced stages (II-IV) of previously untreated HNC. The overall response rate (CR+PR) was $57 \%$ in regimen $A$ and $72 \%$ in regimen $B$. However, the median disease free survival ( 6 vs. 5 mths.) and the median overall survival (12 mths. in both regimens)are similar. Our results suggest that combined modality treatment does not improve treatment results compared to CT alone. Furthermore, responders have only a slight, but significant benefit concerning the survival as compared to nonresponders.Pts. receiving only 2 courses of $C T$ had a similar overall survival compared to those treated with 3 courses of CT or with CT+RT.

Div. of Haematology, Dep. of Internal Medicine, J.W. Goethe University, D-6000 Frankfurt/M, FRG

\section{VHN 11}

Combined Cytostatic(CT)(Vindesine/Platinum) and Radiotherapeutic Treatment(RT) of Squamous Cell Carcinomas (SCC)(UICC IV fof head and neck. SuchyB, KochK, MayrA, JahnkeV, Steinhilber W,OnCol.RVK,D-10000BIn65.0f 171pts.with SCC 28pts.had UICC IV disease.Pts.:age $58 y(35-75) ; 40240 ; n i c o t i n$ and alcohol dependent 26 pts. 10pts. had recurrency, 17 pts. had first manifestation. Planned schedule:2 cycles $(t) \mathrm{CT}$ of Vindesine (VDA) $4 \mathrm{mg} / \mathrm{m}^{2}$ 24hs contin. if. -d1: cDDP $100 \mathrm{mg} / \mathrm{m}^{2} / \mathrm{day} 3 \mathrm{iv..}$ CT were repeated day 21 or 28 releated $\mathrm{H}-\mathrm{Tox}$.. First remission( $R$ )was done after the $t$ 2nd of CT by an interdiscipl. colloquium. If surgeon does not recommend surgical intervention treatment was carried on by combined RT-CT.RT:Single dose of 2,5-3gy twice a week up to total dose of 45-55 gy on tumour area-lymph drainage areas(55gy). Computer tomogr. was conducted in addition to computer assisted RTplanning.During $2 \neq$ of CT was planned additionally.16pts. tb.1 pts. untreated preatr.got $4 \neq \mathrm{CT}, 2$

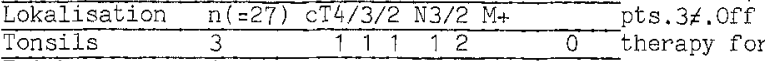

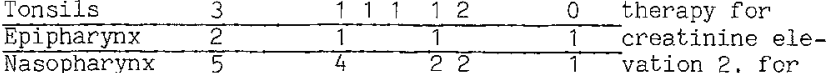

Nasopharynx $5-4-22=1$ vation 2, for

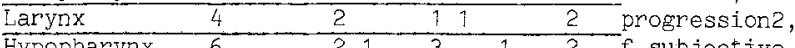
\begin{tabular}{lllllll}
\hline Hypopharynx & 6 & 2 & 3 & 1 & 2 & f. subjective
\end{tabular} Oropharynx 10.10 reasons5pts.

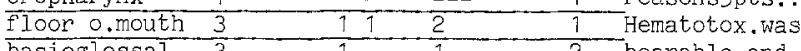
basioglossal $\frac{3}{26} \quad \frac{1}{16}-\frac{1}{16}-\frac{2}{10}$ thearable and

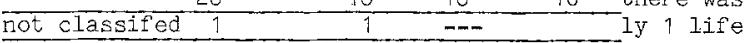
threatening infection with embolization. To Tb.3.: tb.2 Hb $(g / 100 \mathrm{ml}) \quad \mathrm{Lc}\left(\times 10^{3} / \mathrm{ul}\right)$ Plt. $\left(10^{3} / \mathrm{ul}\right) 17 ; \mathrm{pts} . \mathrm{com}-$ Start $13,3(12,0-16,9) 8,5(4,8-13) 282(112-541)$ pleated RT Nadir $10,6(8,7-13,8) 3,13(0,1-6) 136(68-233)$ and simulFirther evaluation after $24 \mathrm{mt} . \mathrm{s}$. $\mathrm{Tb} .3 \quad$ preatr. $(n=10)$ untreat. $(n=17)$ Because of Remission(mts.) 4 mts. $(0-8)-\frac{6}{6.3+(2-16+)}$ partial $R$ Survival (mts.) $5.6+(1-11+) \quad 8.9+(3+-20+)$ gpt s. got dead $\mathrm{n}-4 \quad 58$ seeds 1 pt.got afterloading.Pts, with $\mathrm{R}$.gained 4,7kg(1-9) during therapy. Pts.with PD lost weight.

\section{VHN 12}

FIRST EXPIRIENCE WITH DDP IN COMBINATION WITH 5-FU/VP 16 AS CONTINUOUS VENOUS INFUSION

M. Schroeder, A. Brunöhler (a.G.), L. Leimer (a.G.), H.A. Vaupe1, M. Westerhausen

Continuous venous infusion offers a method of achieving a prolonged plasma concentration of antineoplastic agents with relatively short half lives. Cis-platin has become a widely used antineoplastic agent ( $t / 232$ minutes). The drug administered with a bolus shows typical associated toxicities of acute nausea, vomiting and renal dysfunction.

In animal studies synergistic effects of DDP with 5-FU and VP 16 are known. Since Oct. 1984,62 pts with various forms of cancer were given a five-day continuous venous infusion containing DDP, 5-FU, as well as, VP 16 . Acute nausea und vomiting were drastically reduced. Renal toxicity seems to be reduced also, with retension of neoplastic activity in severalkinds of solide tumors. Loss of electrolytes was also seen. An analysis of our extensive pretreated patients, including toxicity and antineoplastic activity of the chosen regimes, will be presented. More than $50 \%$ of the patients benefited from the treatment, especially patients with Head-and Neck-, Ovarien- and Breast-Cancer.

The good tolerance offers the change for ambulant treatment with cutanous infusion pumps in the near future, how already practised at villejuif.

\section{VHN 13}

INTRAARTERIAL (IA) CHEMOTHERAPY OF STAGE III AND IV SQUAMOUS CELL CARCINOMA OF THE HEAD AND NECK (SCCHN). AN EFFECTIVE APPROACH WITH LOW TOXICITY.

R. Zimmermann, J.v. Scheel, V. Schilling, R. Hermann, E. Kastenbauer

31 patients with previously untreated SCCHN, stage III or IV, without demonstrable distant metastases, underwent radical neck dissection. In the same session, the external carotid artery was prolonged by an autogenic saphenal vein graft and anastomosed more proximally to the common carotid artery, as described elsewhere (J.v. Schee?, Laryng.Rhinol. 60, $275-277,1981$ ). As soon as possible after surgery, cisplatin, $20 \mathrm{mg} /$ day, 3 to 5 days a week, was applied percutaneously into the graft by continuous 8 -hour infusion to a total dose of between 290 and $460 \mathrm{mg}$ After chemotherapy, conventional ly fractionated radiation therapy was given using $\mathrm{Co}-60$. The total dose ranged from 60 to 70 Gy.

The overall response rate was $74 \%$ with $51 \%$ complete and $23 \%$ partial responses. Side effects included nausea, unilateral mucositis and mild myelosuppression. Vomiting was rare. There was one episode of severe infection due to leucocytopenia. Bleeding or lacal infection were not seen. There were 3 cases of temporary elevation of creatinine level (WHO grade I). Hair loss was restricted to the region of IA infusion. All patients completed the regimen.

This pilot study on the feasibility of the above explained treatment programm for locally advanced SCCHN showed a response rate comparable to other recently described scheduTes of aggressive systemic treatment (W.H. Hong, R. Bromer New EngT.J.Med. 308, 75, 1983). In contrast to the regimens of systemic cisplatin application this treatment was remarkabiy well tolerated. It is too early to report on the overall survival time. However, some observed cases of long term survival support the possibility of an improvement of surviva? time and cures in these advanced stages. Medizinische Klinik und HNO-KTinik, Klinikum Charlottenburg der FU, Spandauer Damm 130, D-1000 Berlin 19. 